Comparison of hospitalizations in patients on first generation versus second generation long acting injectable (LAI) antipsychotics

Seemab Rasool ${ }^{\star}$ and Paster Venan

Essex Partnership University NHS Foundation Trust

${ }^{*}$ Corresponding author.

doi: 10.1192/bjo.2021.753

Aims. There is limited data on the comparison of efficacy between first and second antipsychotic LAIs. One good indicator of efficacy is the rates of hospitalization. Some studies have shown that second generation depot antipsychotics, significantly reduce hospitalizations as compared to conventional depots.

Our aim was to compare hospitalizations in patients on first and second generation LAI antipsychotics.

Method. A retrospective observational study was done by reviewing the records of all the depot clinics in South Essex, United Kingdom. A list of patients enrolled and receiving LAI antipsychotics was obtained from the 6 depot clinics. Data were collected by going through the electronic records of the patients on the depot clinic lists and taking down the demographics, diagnosis and the hospital admissions. Other variables like comorbid drug abuse were also recorded.

Result. Amongst a total of 346 patients 223 (64\%) were males and 123 (36\%) were females. Average age was 50.3 (range 21 to 88 years) and $290(83 \%)$ patients were single. An overwhelming majority of patients 299 (87 \%) were not in employment. Regarding the diagnosis, the majority, 237 patients were diagnosed with Paranoid Schizophrenia, 49 patients were diagnosed with Schizoaffective disorder, 38 patients were diagnosed with Bipolar affective disorder, 20 patients had a diagnosis of Delusional disorder and only 2 patients had a primary diagnosis of Mental and Behavioral disorders due to substance abuse. Of the total 346 only 17 patients were on a Community treatment Order.

Risperidone was the most commonly used second generation LAI at $26 \%$,Aripiprazole in $10 \%$ and Paliperidone was used in 5\% patients. Olanzapine LAI was only used in 2 patients. Amongst first generation LAIs Zuclopenthixol, Fluclopentixol were both used in $24 \%$, and Haloperidol in $10 \%$ patients. $21 \%$ of patients were reported to be actively abusing drugs.

$65(32.6 \%)$ of the total 200 patients on Ist Generation LAIs had hospital admissions

55 (39.8\%) of the total 138 patients on 2nd Generation LAIs had hospital admissions

This difference was not statistically significant ( $\mathrm{Z}$ test)- $\mathrm{P}$ value of 0.082427

Conclusion. The results in our observational study are equivocal, both LAIs providing equitable decrease in the hospital admissions albeit with a slightly favourable outcome (not statistically significant though) attributable to the first generation LAIs. There was a high incidence of unemployment and drug abuse in our cohort of patients, thus targeted interventions can be established in rehabilitation of such individuals.

\section{Quantfying the disorganization and the core deficit in} classical schizophrenia

Mohan Rathnaiah ${ }^{1 \star}$, Elizabeth B Liddle ${ }^{2}$, Lauren Gascoyne ${ }^{2}$, Jyothika Kumar², Mohammad Zia Ul-Haq Katshu²,

Catherine Faruqui ${ }^{3}$, Christina Kelly ${ }^{3}$, Malkeet Gill ${ }^{3}$, Sian Robson², Peter Morris $^{2}$, Mathew Brookes ${ }^{2}$ and Peter Liddle ${ }^{2}$

${ }^{1}$ Institute of Mental Health, University of Nottingham; ${ }^{2}$ University of Nottingham and ${ }^{3}$ Nottinghamshire Healthcare NHS Foundation

Trust

${ }^{*}$ Corresponding author.

doi: 10.1192/bjo.2021.754
Aims. To derive scores for mental disorganization and impoverishment from commonly used rating scales, and test the hypothesis that disorganization and impoverishment, along with impaired cognition and role-function reflect a latent variable that is a plausible candidate for the putative core deficit.

Background. For more than 100 years, disorganization and impoverishment of mental activity have been recognised as fundamental symptoms of schizophrenia. These symptoms may reflect a core brain process underlying persisting disability. Delusions and hallucinations have been regarded as accessory features. The psychopathological processes predisposing to persisting disability in schizophrenia are poorly understood. The delineation of a core deficit underlying persisting disability would be potentially of great value in predicting outcome and developing improved treatment.

Method. Patients aged 18-55 years were included if: they satisfied DSM IV criteria for schizophrenia or schizoaffective disorder. Healthy controls were recruited by public advertisement and selected to match the patient group in age and sex. Study sample included 39 participants with schizophrenia, 1 with schizoaffective disorder and 44 matched healthy controls. We derived disorganization and impoverishment scores from three symptom scales: PANSS, SSPI and CASH. We computed composite scores for disorganization and for impoverishment and employed Confirmatory Factor Analysis to test the hypothesis that a single factor accounts for the relationships between disorganization, impoverishment, cognitive impairment and impaired role function. We assessed the relationship between this latent "core deficit" and diminished Post Movement Beta Rebound (PMBR), an electrophysiological measure from Magnetoencephalography (MEG), associated with persisting brain disorders.

Result. Fit indices for the single factor model from CFA indicated a good fit: $\chi 2(2)=1.817, \mathrm{p}=.403$; RMSEA <.001 GFI $=.979$. PMBR was significantly reduced in the schizophrenia group compared to healthy controls, $\mathrm{t}(68)=3.55, \mathrm{p}<.001$. Within the patient group, PMBR was significantly and negatively correlated with the CFA factor scores representing the Core Deficit score, $\mathrm{r}=-.543, \mathrm{p}<.01$, indicating that high core deficit scores were associated with reduced PMBR. PMBR was significantly correlated with the composite Disorganization score, $\mathrm{r}=-.521, \mathrm{p}<.001$.

Conclusion. Our findings demonstrate that the shared variance between impoverishment (psychomotor poverty); disorganization; cognitive impairment; and impaired role function can be accounted for by a latent variable that can reasonably be described as the core deficit of classical schizophrenia. The demonstration that the severity of the putative core deficit is correlated with the reduction in PMBR provides evidence that the core deficit is associated with an identifiable abnormality of brain dysfunction.

Prevalence of mental disorders in prisons in the UK: a systematic review and meta-analysis

Nivedita Rebbapragada*, Vivek Furtado and

George William Hawker-Bond

Warwick Medical School

${ }^{\star}$ Corresponding author.

doi: 10.1192/bjo.2021.755

Aims. To report pooled prevalence of all mental disorders among the general prison population in the United Kingdom (UK). This includes individuals in Young Offender Institutions (YOI), youth custody and adult prisons across all categories. A secondary aim explores possible sources of heterogeneity by performing 